Session 2259

\title{
Distributed Control Systems in the Process Control Laboratory
}

\author{
James A. Rehg, Dr. Peter J. Shull \\ Penn State Altoona
}

\begin{abstract}
Industrial process control, which moved from Direct Digital Control (DDC) to Distributed Control Systems (DCS) in the late 1970s, is now making another transition to Field Control Systems (FCS). While FCS is just a form of DCS, it adds additional dimensions to the control function through the use of networked systems and smart control elements. Foundation Fieldbus (FF), an implementation of FCS based on international standards, was introduced in 1994 and is becoming an established technology for use in industrial control systems. This paper describes the development of a FCS process control laboratory that includes FF and other networked software products to enhance the learning in a control based laboratory. In addition, the paper compares process control issues using the older DCS model with the Fieldbus solution, and it describes the hardware and software used at Penn State Altoona to build a fully networked FCS process laboratory.

Introduction

Foundation fieldbus is a digital control network that inter-links "smart" sensors and actuators in a manufacturing environment. It is one of the latest technologies used to automate the capture of process data and the control of production systems. The evolution of the system architecture from Direct Digital Control (DDC) to Distributed Control Systems (DCS) and now to Field Control Systems (FCS) is illustrated in Figure 1. The yaxis indicates when the normal distribution of the adoption of the different system architectures peaked in use. In every step of the evolution, the control of the process has moved closer to the sensors and actuators.
\end{abstract}


Figure 2 illustrates the shift of the proportional-integral-differential (PID) function from the primary system computer to the sensors and actuators at the point of measurement and control. This movement of the control process, while more costly initially, reduces wiring, aids in troubleshooting, and

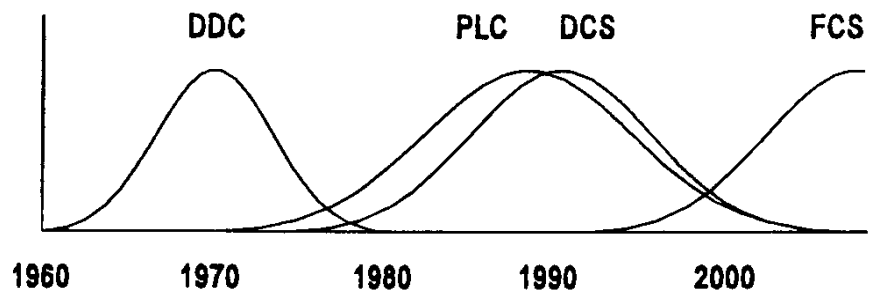
decreases maintenance costs of the industrial control network. It also allows fieldbus devices to be controlled by any host computer with the appropriate interface to the fieldbus system, which is on the plant Local Area Network (LAN).

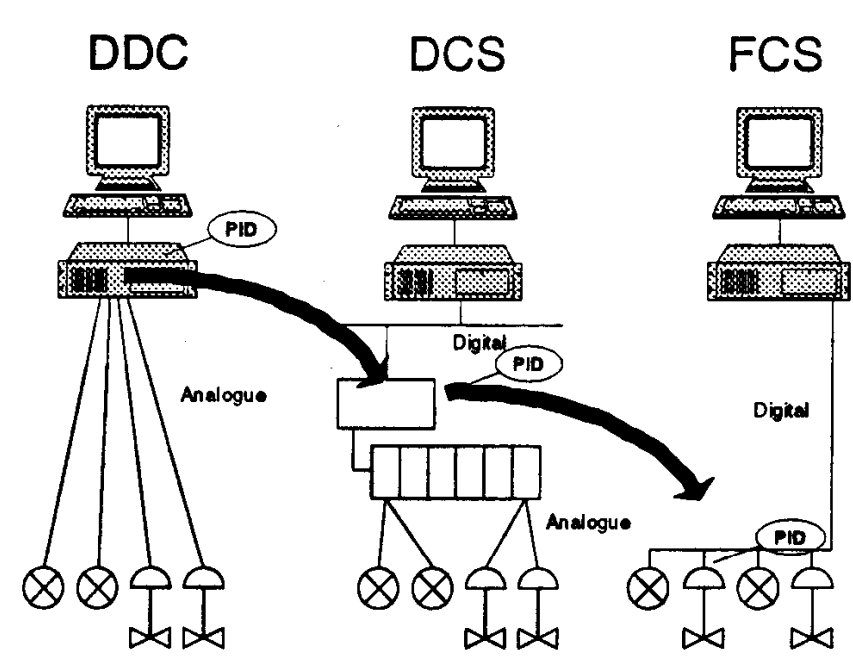

Figure 2 Control Architecture [1]

Another feature of the FF system is the capability of adding fieldbus devices to an existing fieldbus process while it is operational. This makes the implementation of new fieldbus networks into an existing system a less complex process and does not require that the process be shutdown when sensors are replaced or new field devices are added. Fieldbus devices have the ability to run process control loops internally without having any need for processing power from a central computer or digital processor on the network. In addition to the data networking function, the FF twistedpair network cable can supply the power required to run all the sensors and actuators on the network. The standard stipulates that up to 32 devices can be placed on a single segment. Repeaters permit up to 240 fieldbus devices on a single network.

The FF standard of interoperability supports "Plug and Play" architecture. This allows field devices from different vendors to be mixed in a working fieldbus model and the addition of new field devices without the need for major LAN reconfiguration.

\section{Fieldbus Systems and Standards}

The number of network control techniques, using the term fieldbus to describe their operation, is numerous. As a result, a considerable level of confusion exists in the selection and design of a fieldbus driven control system. The chart in Figure 3 provides an overview 
of the current network protocol

choices available to the design engineer. The fieldbus LANs are divided into the two broad application categories of discrete and process. The level of automation available further differentiates the choices from bit-level sensor operation to support for the process control and interfaces into the business unit for database management and inventory control. In the discrete area, the Profibus DP, CAN, ControlNet, DeviceNet, and SDS protocols have

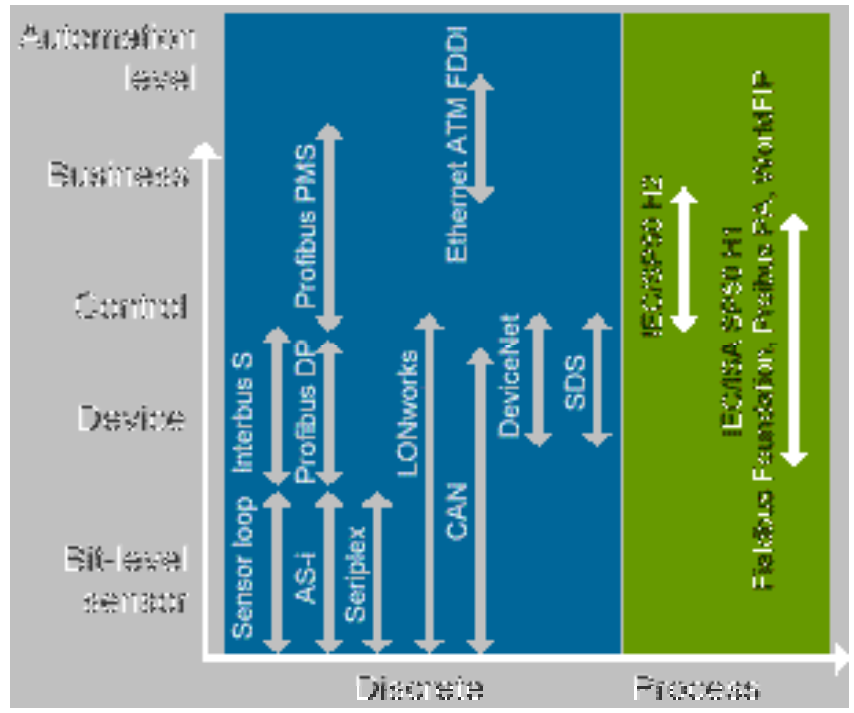

Figure 3 Fieldbus LAN Options [2] good vendor support. On the process control side, two protocols predominate: the Fieldbus Foundation and Profibus PA. Development of the fieldbus standard started in the mid-1980s when the Instrument Society of America (ISA) formed the SP50 fieldbus committee. In 1992, the number of variations in the standard narrowed when Fisher, Rosemount, Yokogawa, and Siemens created the Inter-operable Systems Project (ISP) and the other major SP50 companies, including Honeywell, Allen Bradley, and others formed the WorldFIP standard group [2]. Further consoli-dation occurred in 1993 when the ISP and WorldFIP

joined to form the Fieldbus Foundation (FF). As a result, two protocols have evolved for LAN based process control applications: the Fieldbus Foundation, a standard supported in the United States and Asia, while the Profibus PA standard is popular in Europe.

\section{Fieldbus Architecture at Penn State Altoona}

The Foundation Fieldbus (FF) architecture used in the Penn State Altoona (PSA) process control engineering laboratory is illustrated in Figure 4. The system was purchased through an NSF Laboratory Instrumentation Grant at a cost of $\$ 32,000$. The college received significant discounts from the vendor to support this laboratory upgrade project. The PSA system from Emerson Process Management uses hardware from Fisher-Rosemont and the DeltaV control software. The system includes a central control station and three remote process control stations. The process trainer, supported at the

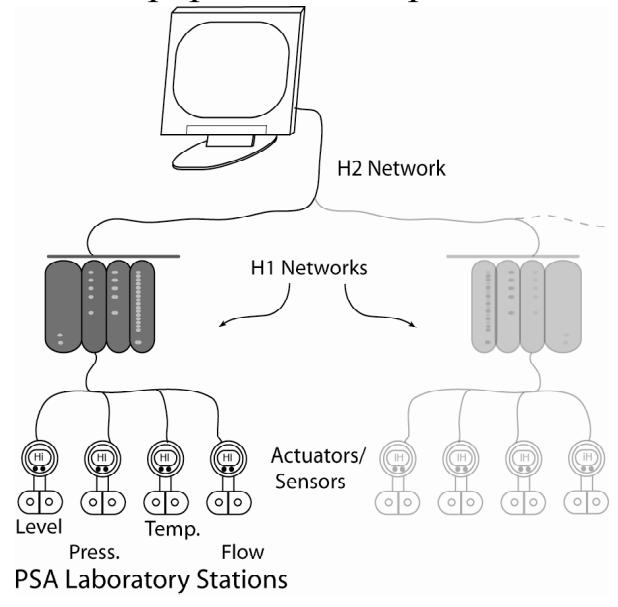

Figure 4 Fieldbus configuration used at PSA central station, is a flow and/or level type system with a pressure, level, and temperature 
trainer at the three remote stations.

The PSA system in Figure 4 is configured with two LAN types, called H1 and H2. The system illustrated in Figure 5 shows the four methods used to attach field devices to the H1 LAN. The H1 segment is a $31.25-\mathrm{kbit} / \mathrm{sec}$ bus structure used to link FF devices together. The $\mathrm{H} 1$ bus, illustrated in Figure 4 and 5, can be point-to-point, bus with spurs or multi-drop, daisy chain, and tree. Type A shielded twisted pair wire is used for the H1

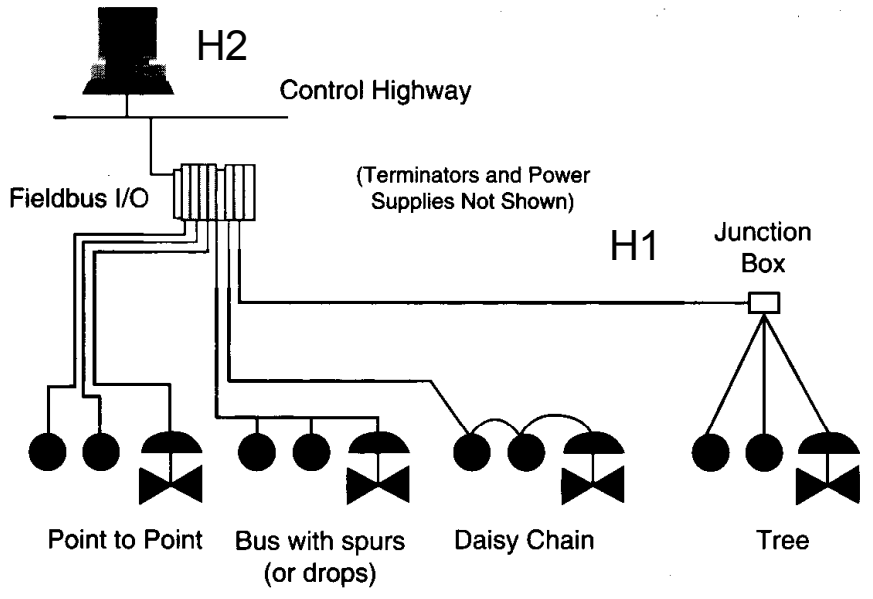

Figure 5 Possible Fieldbus Topologies [3] connections as specified in the IEC/ISA physical layer standard [3]. A maximum length of 1900 meters for $\mathrm{H} 1$ cabling is also specified in the same standard. While new installations would use this wire, most current implementations could convert to FF technology using existing instrumentation wiring in most situations. In the PSA implementation the input/output $(\mathrm{I} / \mathrm{O})$ rack is located at the central computer station with the three remote stations linked by an $\mathrm{H} 1$ LAN to the central control. The

link between the $\mathrm{I} / \mathrm{O}$ and the central computer is an $\mathrm{H} 2 \mathrm{LAN}$.

This second FF LAN, called $\mathrm{H} 2$, is a high-speed fieldbus communications mode, which serves as a backbone for the $\mathrm{H} 1$ segments. The $\mathrm{H} 2$ backbone can operate at 1, 2.5, or $100 \mathrm{Mbits} / \mathrm{s}$. The $\mathrm{H} 2$ network speeds are useful for transferring data between the smart field devices and other production hardware like programmable logic controllers (PLCs) and process analyzers. The $\mathrm{H} 2$ LAN permits access to the fieldbus structure from any computer on a plant intranet, and gives process engineers and production planners direct access to process data and the ability to program the system from remote locations.

\section{Laboratory Software Implementation}

The process control laboratory at PSA supports laboratories for two classes: EMET 330 Measurement Theory and Instrumentation and EMET 410 Automatic System Controls. In the first class, EMET 330, students use a combination of small trainers and individual sensors and actuators to learn such topics as calibration, hysteresis measurement, setting of span and zero, determination of dynamic range and noise sensitivity. In each experiment students use the DeltaV software to acquire the sensor and actuator data.

In the second course, EMET 410, students are introduced to PID process control with proportional, $\mathrm{P}$, used for initial control, then integral, I, is added for PI control, and finally derivative, D, is added for full PID control exercises. The DeltaV software is used for control and operation, but INTUNE, a stand-alone tuning software, is used for simulation of exercises and determination of tuning parameters. In addition, integrating and non-integrating process loops are tested; long deadtime processes are implemented 
using IMC control schemes with feed forward logic.

The INTUNE software is used because the DeltaV supports tuning at the central station but not at each remote station, so INTUNE provides that function along with simulation capability. An example of the tuning process is shown in Figure 6 (PVprocess variable, $\mathrm{CO}$-controller output, SP-set point). The pressure system is initially poorly tuned. Using INTUNE, a closed loop setpoint bump test is used to calculated new PID parameters. These new tuned PID values are then downloaded to the system with response as shown in the lower chart in Figure 6.

\section{Introduction of Fieldbus into the Laboratory}

The first step in creating a Foundation fieldbus system is to select the process to control. Implementations of the fieldbus network in the control laboratory can include a number of process systems, both new and existing. Most process control laboratories in colleges and universities have process trainers for teaching control of temperature, pressure, flow, and level. A typical flow trainer in the PSA laboratory is shown in Figure 7. The 4-20 $\mathrm{mA}$ control systems and standalone PID controllers in these simulators can be supplemented by fieldbus devices without losing the 4-20 ma control option. This is the technique used in the PSA implementation of the fieldbus process laboratory.

Fieldbus sensors and actuators are available from a number of instrumentation vendors. The basic components are temperature sensors, pressure sensors, flow sensors, level sensors, valve positioners, pneumatic
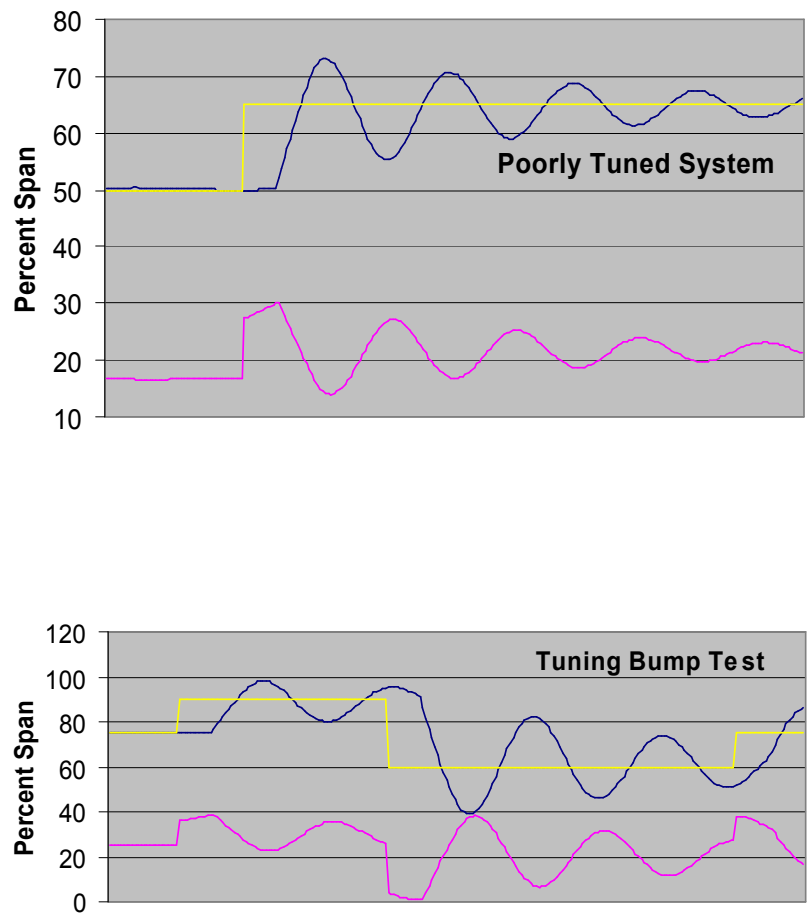
valve blocks, fieldbus-to-current (4 to $20 \mathrm{~mA}$ ) converters, and current (4 to $20 \mathrm{~mA}$ )-tofieldbus converters. Additionally, "Round Cards" are available to connect existing 4-20 $\mathrm{mA}$ devices into Fieldbus networks. 


\section{Modifying Existing Trainers}

The fieldbus network and control system, designed for the process control laboratory at Penn State Altoona, utilizes four existing process trainer like the one shown in Figure 7. The fieldbus devices are integrated into the existing 420 ma loop control system. The fieldbus sensors, a differential sensor in Figure 8 for the flow system, are placed into the systems so that operation with standard 4-20 ma loop using standard process sensors and actuators can be compared to control using fieldbus measurement devices. The sensor illustrated in Figure 8 is a differential pressure type device.

In the fieldbus solution the system operation is configured and controlled using the DeltaV distributed network with the PID controller located in the fieldbus field device. The H1 network shown in Figure 4 illustrates the PSA implementation except that a bus with spurs or drops is used for each of the trainer locations. When standard control components are used, a Foxboro single loop controller located on the trainer performs the control. When standard components are used for control, the DeltaV

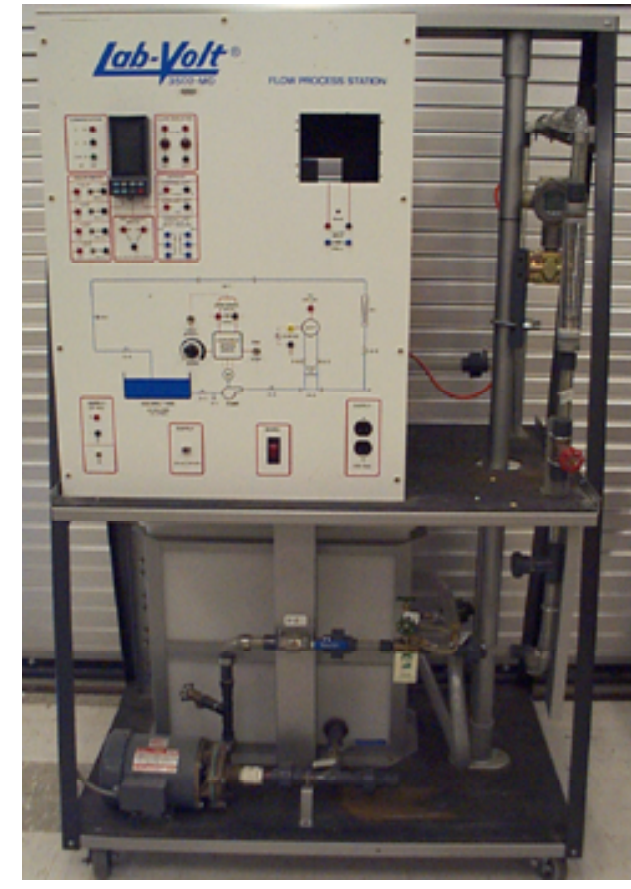

Figure 7 Lab-Volt Flow Trainer system is used to measure and display the system performance. As a result, the performance of the fieldbus and standard solution can be compared.

\section{Teaching Advantages and Disadvantages}

The particular implementation of both a fully automated control system and a manual one creates teaching advantages on two levels. First, the dual system offers some learning advantages by focusing on elements of each system, and second, the redundant system allows for backup in the case where software issues threaten to diminish the function of a particular laboratory assignment.

When teaching instrumentation or controls or any laboratory, pedagogically it is important to begin a concept without the complexities often associated with sophisticated software. Consequently many ideas are introduced using the manual system. Additionally, at times automated systems mask the fundamentals. For example, when calibrating a sensor, the functional relationships between zero and span are evident if the sensor is calibrated manually. On the other hand, this relationship is completely lost in an automated calibration.

In contrast, the industrial, automated system generally reduces the configuration, operation, and data acquisition time. This allows the students more learning opportunities such as easier data collection, reduction, and analysis as well as opportunity to observe 
system responses to variation of parameters such as PID values. Additionally, the students experience working with complex industrial systems.

Probably the biggest disadvantage of the DeltaV-Fieldbus system is its complexity. If students become lost, there is a tendency to just try things. Under this scenario, the program configuration can become very difficult to correct quickly enough to accommodate a laboratory setting. Thus the students become frustrated with the assignment. Additionally as the equipment is not designed specifically for teaching, issues

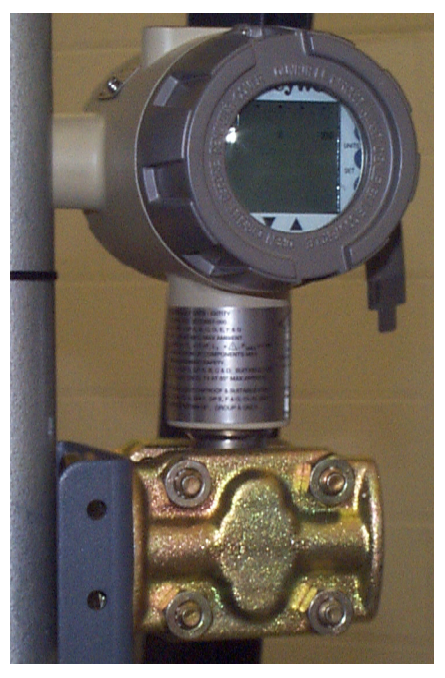
such as how to teach the concepts without too much focus on the DeltaV program details can be difficult to resolve.

\section{Conclusions}

Fieldbus is beginning to find a broad use in manufacturing control for the following reasons: it is relatively easy to reduce costs by allowing a user to start with a small system and expand as necessary and financial abilities allow, field wiring is reduced, and troubleshooting of system problems is enhanced. Also, since Foundation fieldbus components are compatible between companies, a number of vendors have created components for use in Foundation fieldbus systems in industry, allowing for greater competition in the market.

Because of the anticipated benefits of using a

Figure 8 - Fieldbus

Differential Pressure

Transmitter

Foundation fieldbus system, it is important to teach

Foundation fieldbus in the educational environment. The education of future control engineers in this new technology is the key to moving process control from the distributed control system model to the networked model described by Foundation fieldbus.

\section{Acknowledgements}

We gratefully acknowledge the generous contributions in both equipment and assistance from ControlSoft (INTUNE) and Emerson (Rosemount and Micromotion). We would like to thank Philip B. Szajnuk Jr., Michael Mihuc, and Lance Baum from Rosemount and Graham England and Paul Botzman of ControlSoft This work was partially supported by NSF under grant number 0127013.

\section{Biographies}

James Rehg is an associate professor and Program Coor-dinator of the B. S. program in Electromechanical Engin-eering Technology at Penn State Altoona. Dr. Peter J. Shull is an associate professor and Program Coordinator of the A. S. program in Mechanical En gineering Technology at Penn State Altoona. 


\section{References}

1. Berge, J., "Addressing Benefits and FAQs of Fieldbused FCS Architecture", Instrument Society of America, Publication number 10

2. Studebaker, P., "Fieldbus: Reaching the Promised LAN", Control Engineering, April 1997, http://www.controlmagazine.com/0497/c0260497.html.

3. Glanzer, D., Wiring and Installation $31.25 \mathrm{kbits} / \mathrm{s}$, Voltage Mode, Wire Medium - Application Guide, Fieldbus Foundation, 1996, pp. $14-16$.

4. McDougall, S., I. Verhappen, S. Wheatman, "Fieldbus Testing - Putting an Alliance to Work", Instrument Society of America, Publication number 5.

5. Glanzer, D., "Foundation fieldbus and its Role in the Plant Network Hierarchy", Instrument Society of America, Publication number 20.

6. Hendricks, D., http://www.barn.org/FILES/historyofplc. html, R. Morley Incorporated.

7. Hodson, W., "Fieldbus to Change DCS Role, but Death Reports Greatly Exaggerated", Honeywell, 1998.

8. $\quad$ Fieldbus Wiring and Installation Guide, Relcom, Inc., www.relcominc.com.

9. Wiring and Installation Application Guide, Fieldbus Foundation, www.fieldbus.org/information/ 